\title{
Comparaison des prévisions à court et à long terme d'un modèle de croissance à l'échelle du peuplement avec celles d'un modèle à l'échelle de l'arbre
}

\author{
par Hugues Power ${ }^{1}$ et Isabelle Auger ${ }^{2}$
}

\begin{abstract}
RÉSUMÉ
Différents modèles de croissance forestière sont aujourd'hui disponibles pour simuler lévolution des peuplements forestiers du Québec. Pour choisir le modèle qui répond le mieux à ses besoins, l'utilisateur a donc besoin d’informations. La présente étude a pour but dévaluer et de comparer le comportement de deux modèles de croissance forestière évoluant à des échelles de résolution différentes : Artémis, un modèle à léchelle de l'arbre, et Natura, un modèle à léchelle du peuplement. Pour ce faire, nous avons utilisé les deux modèles pour simuler lévolution sur une période de 50 ans de placetteséchantillons permanentes regroupées en strates et réparties sur 6 larges territoires de la forêt publique québécoise. Nos résultats montrent que les deux modèles produisent des résultats cohérents, mais que leur performance varie fortement selon le territoire et la strate simulés. Les résultats nous portent à recommander l'utilisation de Natura sur les portions de territoire situées dans le domaine bioclimatique de la pessière à mousses et majoritairement composées dépinettes noires (Picea mariana [Mill.] B.S.P.), et à privilégier Artémis pour les peuplements dont la structure est plus complexe qui sont situés dans les domaines de la sapinière à bouleau blanc et de la sapinière à bouleau jaune.
\end{abstract}

Mots-clés : modèles de croissance forestière, aménagement forestier, échelle de résolution, tordeuse des bourgeons de lépinette, Artémis, Natura, possibilité forestière

\section{ABSTRACT}

During the past decades, many growth models have been developed that can now be applied over large parts of Québec's forest. For the user, choosing the right model for a given territory of interest can be challenging. The present study aims to compare two forest growth models which can be used over most of Québec's commercial forest: Artémis, a tree-level model, and Natura, a stand-level model. To compare the two models, we used measures from permanent sample plots located in six distinct territories across the province of Québec (Canada). Each plot was simulated with each growth model for a period of 50 years. Both models produced coherent forecasts, but their precision and accuracy varied among groups of plots and territories. Overall, Natura had a better performance than Artémis in the spruce-moss bioclimatic domain, while Artémis performed better in the balsam fir-white birch and the yellow birch-balsam fir domains.

Key words : forest growth models, forest management, tree level models, Artémis, stand level models, Natura

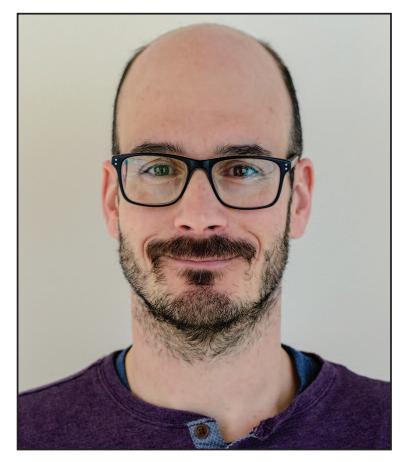

Hugues Power

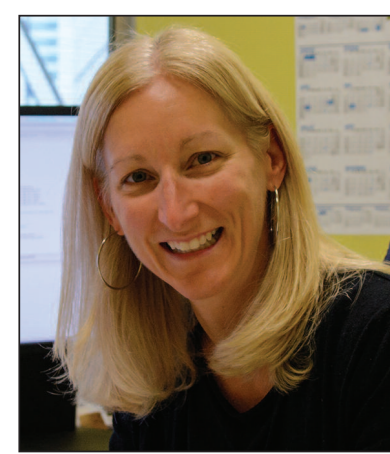

Isabelle Auger

\section{Introduction}

La prévision de lévolution des caractéristiques des peuplements forestiers suscite l'intérêt des forestiers depuis plusieurs décennies. La création des tables de production en Europe, à la fin du $18^{\mathrm{e}}$ siècle, figure parmi les premiers outils permettant d'estimer les caractéristiques futures d'une forêt (Pretzsch $e t$ al. 2008). Au fil du temps, les méthodes destimation se sont raffinées et ont évolué vers des modèles de croissance dont l'utilisation s'est accrue au cours des dernières décennies (Larocque et al. 2009). Ceux-ci sont considérés comme plus flexibles que les tables de production, puisqu'ils permettent de faire varier les conditions de croissance d'un arbre ou d'un peuplement dans le temps, et donc d'obtenir des prévisions plus réalistes (Stage 2003).

\footnotetext{
${ }^{1}$ Auteur chargé de correspondance : Direction de la recherche forestière, ministère des Forêts, de la Faune et des Parcs, 2700, rue Einstein, Québec (Québec) G1P 3W8, Canada. Courriel : hugues.power@mffp.gouv.qc.ca.

${ }^{2}$ Direction de la recherche forestière, ministère des Forêts, de la Faune et des Parcs, 2700, rue Einstein, Québec (Québec), G1P 3W8, Canada.
} 
Compte tenu des différents besoins des utilisateurs, des modèles de croissance ont été mis au point à différentes échelles de résolution. Les modèles à l'échelle de l'arbre et du peuplement sont aujourd'hui les plus utilisés en foresterie (Burkhart 2003). Plusieurs auteurs ont apporté leurs commentaires sur l'échelle de résolution optimale des modèles de croissance. Pour certains, les modèles devraient faire des prévisions directement à léchelle à laquelle ils sont utilisés (Landsberg 2003). Pour d'autres, plus l'horizon de simulation est lointain, plus on doit diminuer le niveau de résolution du modèle (Burkhart 2003). Même si les modèles à l'échelle du peuplement peuvent être robustes, leurs limites sont parfois atteintes lorsqu'il s'agit de simuler des interventions sylvicoles ou des peuplements de structure complexe (Groot et al. 2004). En contrepartie, les modèles à l'échelle de l'arbre peuvent parfois difficilement prévoir les caractéristiques du peuplement, puisqu'ils nont pas été paramétrés pour prévoir directement ces variables réponses (Vospernick et al. 2015). Par conséquent, certains auteurs suggèrent d'utiliser les modèles à l'échelle de l'arbre pour les prévisions à court terme afin de profiter de leur niveau de détail, et d'utiliser les modèles à léchelle du peuplement pour les prévisions à plus long terme afin de profiter de leur robustesse (Mäkinen et al. 2008). D’autres auteurs ont combiné les deux échelles en utilisant les prévisions d'un modèle à l'échelle du peuplement afin d'ajuster celles d'un modèle à l'échelle de l'arbre (ex. Yue et al. 2008). Peu importe le modèle choisi ou sa résolution, il est important de soumettre celui-ci à un processus d'évaluation continu afin de bien documenter son comportement dans différentes conditions d'utilisation (Vanclay et Skovsgaard 1997).

En 2009, afin de soutenir l'aménagement forestier au Québec, notamment dans le cadre du calcul des possibilités forestières, la Direction de la recherche forestière (DRF) du ministère des Forêts, de la Faune et des Parcs (MFFP) a mis au point un modèle de croissance à léchelle du peuplement (Natura2009; Pothier et Auger 2011) et un modèle de croissance à léchelle de l'arbre (Artémis-2009; Fortin et Langevin 2010). Les deux modèles ont été paramétrés avec des données provenant de placettes-échantillons permanentes (PEP) du réseau de la Direction des inventaires forestiers (DIF) du MFFP et peuvent être appliqués à l'ensemble de la forêt commerciale du Québec. Comme les modèles Artémis et Natura fonctionnent à des échelles de résolution différentes, ils produisent aussi des informations différentes. Natura produit une estimation de la densité des arbres marchands, de la surface terrière marchande, du volume marchand brut, du diamètre moyen quadratique et du volume moyen par tige, et ce, tant pour le peuplement en entier que pour 5 groupes d'essences pris séparément (Pothier et Auger 2011). Artémis, quant à lui, génère des prévisions à l'échelle de l'arbre à partir desquelles on peut dériver l'ensemble des extrants générés par Natura. De plus, contrairement à Natura, Artémis permet d’obtenir de l'information détaillée sur la composition en essences, la distribution diamétrale et le rendement en produits lorsque les sorties du modèle sont jumelées à un simulateur de débitage. Artémis permet également de simuler les effets d'interventions sylvicoles. Les deux modèles comportent une fonctionnalité leur permettant de simuler le comportement des peuplements forestiers en période dépidémie de tordeuse des bourgeons de l'épinette (TBE) (Choristoneura fumiferana [Clemens]). Cette particularité est essentielle, puisque la forêt boréale québécoise subit périodiquement des dommages par cet insecte défoliateur (Gray et al. 2000).

En 2013, avec la progression du $4^{\mathrm{e}}$ inventaire écoforestier du Québec, les modèles Artémis et Natura ont été paramétrés de nouveau. Les nouvelles versions des modèles ont permis d'ajouter près de 4000 intervalles de croissance pour Natura (Auger 2017a) et près de 6000 pour Artémis (Power 2016). Cela représente des augmentations respectives de $30 \%$ et de $25 \%$ du jeu de données de base qui avait servi à paramétrer les modèles en 2009.

Comme les deux modèles peuvent s'appliquer sur les mêmes territoires, l'utilisateur doit choisir lequel utiliser en fonction des objectifs poursuivis par ses travaux. La présente étude vise donc, premièrement, à comparer le biais et l'exactitude des prévisions à court terme ainsi que les valeurs des prévisions à long terme d'un modèle de croissance à l'échelle de l'arbre, Artémis, et d'un modèle de croissance à l'échelle du peuplement, Natura, dans différentes régions du Québec, pour des groupes de peuplements qui diffèrent par leur composition en essences, leur âge, leur densité et leur végétation potentielle. Deuxièmement, létude vise à mettre en évidence des indications qui permettraient à l'utilisateur de choisir le modèle à utiliser selon les conditions d'utilisation.

\section{Matériel et Méthodes \\ Modèles de croissance}

La version 2014 du modèle Artémis a été utilisée dans le cadre de cette étude. Pour chacune des tiges de plus de 9,0 cm de diamètre à hauteur de poitrine (DHP; mesuré à une hauteur de $1,3 \mathrm{~m}$ ) comprise dans une placette de $400 \mathrm{~m}^{2}$, le modèle prévoit la probabilité de mortalité ainsi que l'accroissement du DHP en cas de survie. Artémis inclut un module de recrutement qui permet au modèle de générer de nouvelles tiges de plus de $9 \mathrm{~cm}$ de DHP pendant toute l'évolution du peuplement. Le modèle fonctionne par périodes de simulation de 10 ans, et les résultats de chacune des périodes sont réutilisés par le modèle comme point de départ pour la période de simulation suivante. Artémis fonctionne en mode stochastique ou en mode déterministe. Pour les besoins de comparaison avec Natura, qui fonctionne seulement en mode déterministe, nous avons utilisé le mode déterministe d'Artémis pour réaliser cette étude. Le fonctionnement détaillé du modèle Artémis est décrit dans Fortin et Langevin (2010) et dans Power (2016).

La version 2014 de Natura prend comme point de départ une table de peuplement à partir de laquelle il calcule le nombre de tiges marchandes, la surface terrière marchande et le volume marchand brut dans une placette de $400 \mathrm{~m}^{2}$. Ces calculs sont faits pour cinq groupes d'essences : les feuillus intolérants à l'ombre (FI), les feuillus tolérants à l'ombre (FT), les résineux tolérants à lombre (RT), les résineux intolérants à lombre (RI) et le sapin baumier (Abies balsamea [L.] Mill.). Une liste détaillée de la composition des groupes d'essences peut être trouvée à l'Annexe 1 de Auger (2017a). Par la suite, Natura fait évoluer les caractéristiques dendrométriques de chacun des groupes d'essences. Tout comme Artémis, Natura fonctionne avec des périodes de simulation de 10 ans; les prévisions de chacune de ces périodes sont réintroduites dans le 
Tableau 1. Caractéristiques des territoires de simulation

\begin{tabular}{|c|c|c|c|}
\hline Territoire de simulation & $\begin{array}{l}\text { Unités d'aménagement } \\
\text { forestier (UAF) }\end{array}$ & $\begin{array}{c}\text { Mesures de placettes-échantillons } \\
\text { permanentes (PEP) }\end{array}$ & $\begin{array}{l}\text { Période couverte par } \\
\text { les simulations }\end{array}$ \\
\hline Nord-Ouest & $\begin{array}{c}08551,08652,08751,08763,08762 \\
08764,08666,08665,08664,08663\end{array}$ & 239 & $1972-2012$ \\
\hline Nord-Est & 09351, 09451 & 164 & 1974-2002 \\
\hline Ouest & $08651,08451,08462$ & 149 & $1972-2007$ \\
\hline Centre & $03153,02351,02352,03351$ & 178 & 1971-2007 \\
\hline Est & $11161,11262,11263$ & 114 & 1971-2005 \\
\hline Sud-Ouest & 07451 & 225 & $1972-2002$ \\
\hline
\end{tabular}

modèle comme conditions initiales de la période de simulation suivante. Le fonctionnement de Natura est décrit dans Pothier et Auger (2011) et dans Auger (2017b).

Les modèles Natura et Artémis sont programmés en langage Java sur la plateforme de simulation Capsis (DufourKowalski et al. 2012). Ils peuvent être téléchargés gratuitement à l'adresse suivante : ftp://ftp.mrn.gouv.qc.ca/public/ drf/capsis.

\section{Données}

Afin de comparer les comportements des modèles de croissance, nous avons utilisé un jeu de données constitué des mesures des PEP. Ces placettes-échantillons circulaires d'une dimension de $400 \mathrm{~m}^{2}$ ont été établies à partir du milieu des années 1970 et couvrent l'ensemble de la forêt sous aménagement forestier au Québec. Tous les arbres de plus de $9 \mathrm{~cm}$ de DHP sont numérotés et sont mesurés à une fréquence moyenne de 10 ans (MFFP 2014). Nous avons retiré du jeu de données les mesures pour lesquelles une coupe partielle ou une coupe totale avait été effectuée durant la période d’observation puisque la comparaison entre les valeurs mesurées dans les PEP et les valeurs simulées était alors impossible à effectuer. Afin d'évaluer les modèles Artémis et Natura selon leur échelle habituelle d'utilisation sur le territoire de la forêt publique québécoise, nous avons sélectionné des territoires sur la base de leur appartenance à une unité d'aménagement forestier (UAF; Poulin 2013a). Nous avons regroupé des UAF sur la base de leur emplacement géographique afin de créer des territoires de simulation. En tout, nous avons sélectionné 6 territoires regroupant 23 UAF et 1120 mesures de PEP (Tableau 1, Fig. 1).

Dans chacun des six territoires, nous avons regroupé les mesures de PEP pour créer des groupes homogènes possédant des caractéristiques similaires (c. à d. les strates regroupées) en vue de simuler leur croissance. Le regroupement, basé sur l'appellation cartographique des peuplements lors de la mesure des PEP, s'est fait selon des critères semblables à ceux utilisés par le Bureau du forestier en chef (BFEC) lors du calcul des possibilités forestières (Poulin 2013b). Nous avons choisi la strate regroupée comme échelle de résolution afin de comparer le comportement des modèles à une échelle proche de leur contexte habituel d'utilisation. Afin de constituer ces strates regroupées, nous avons rassemblé les mesures de PEP sur la base de leur essence principale, leur classe de densité du couvert, leur classe d'âge et leur végétation potentielle (Tableau 2). Pour que les critères de regroupement se rapprochent de ceux utilisés par la BFEC, nous navons retenu que les strates qui comptaient au moins 10 mesures de PEP et qui avaient une précision sur le volume total d'au moins $60 \%$ (Eq. 1) :

$$
\text { [1] } P=100-\left(\frac{(\sigma * t)}{\bar{v}} * 100\right)
$$

où $P$ est la précision du volume marchand brut de la strate, toutes essences confondues, $\sigma$ est l'erreur type du volume marchand brut pour la strate, toutes essences confondues, $t$ est la valeur Student pour une probabilité de 0,025 et $\bar{V}$ est le volume marchand brut moyen de la strate, toutes essences confondues.

Après avoir créé les 44 strates regroupées (Tableau 3), nous avons utilisé les modèles de croissance Artémis et Natura pour simuler lévolution de chacune des placettes composant les strates sur une période de 50 ans. Une seconde simulation a été effectuée en supposant une défoliation grave causée par la TBE lors de la première période de simulation (10 premières années). Pour Artémis, la simulation de lépidémie de TBE se base sur l'identification des intervalles de croissance des PEP pour lesquels il y avait quatre années consécutives ou plus de défoliations modérées ou graves (Fortin et Langevin 2010). Natura, quant à lui, utilise l'indice de réduction de croissance associé à l'occurrence d'épidémie de TBE de Pothier et Mailly (2006). Dans le cadre de cette étude, nous avons utilisé la valeur maximale de l'indice de réduction de croissance (Auger 2017b).

\section{Évaluation et comparaison des prévisions des modèles}

Nous avons effectué une première évaluation des modèles en comparant les prévisions après une période de simulation aux observations correspondantes des mesures des PEP. Pour ce faire, nous avons utilisé la mesure de chacune des PEP suivant celle utilisée pour amorcer la simulation comme observation de référence. Étant donné que la durée d'une période de simu- 


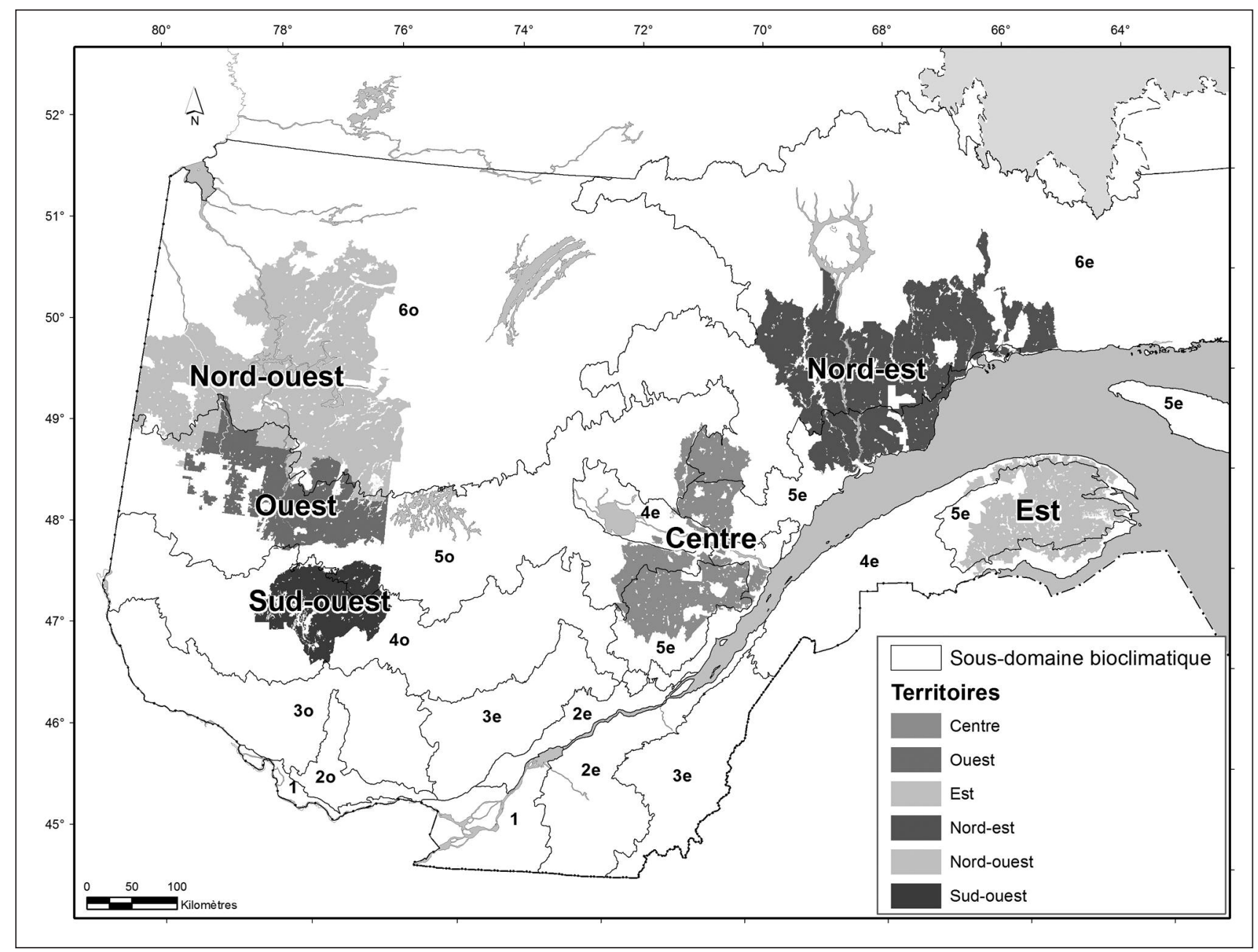

Fig. 1. Territoires retenus pour cette étude

Tableau 2. Liste des critères de regroupement et contenu des strates regroupées

Critères de regroupement Code du groupe et sa définition

Essence principale cartographiée

Classe de densité du couvert cartographiée

Code de végétation potentielle cartographié

Classe d’âge cartographiée
$\mathrm{Bj}$ : bouleau jaune (Betula alleganiensis Britt.)

$\mathrm{Bp}$ : bouleau à papier (Betula papiryfera Marsh.)

Ep : épinette noire (Picea mariana Mill.) et épinette rouge (Picea rubens Sarg.)

$\mathrm{Fi}$ : feuillus intolérants

Fx : autres feuillus

$\mathrm{Pg}$ : pin gris (Pinus banksiana Lamb.)

$\mathrm{Sb}$ : sapin baumier (Abies balsamea Mill.) et épinette blanche (Picea glauca Moench.)

Fa : faible (25-60\%)

Fo : forte $(61-100 \%)$

MJ1 : bétulaie jaune à sapin et érable à sucre

MJ2 : bétulaie jaune à sapin

MS1 : sapinière à bouleau jaune

MS2 : sapinière à bouleau blanc

RE2 : pessière noire à mousses ou à éricacées

$\mathrm{RE} 3$ : pessière noire à sphaignes

RS2 : sapinière à épinette noire

Je : jeune (30, 50, 70, jeune de structure inéquienne)

$\mathrm{Ma}:$ mature $(90,120$, vieille de structure inéquienne) 
Tableau 3. Caractéristiques moyennes des mesurages des placettes-échantillons sélectionnées pour l'étude, par territoire de simulation et strate regroupée (minimum-maximum entre parenthèses). Voir le tableau 2 pour la définition des codes utilisés dans le nom des strates regroupées (Fi : feuillus intolérants, Ft : feuillus tolérants, Ri : résineux intolérants, Rt : résineux tolérants, Sb : sapin baumier].

\begin{tabular}{|c|c|c|c|c|c|}
\hline $\begin{array}{l}\text { Territoire de } \\
\text { simulation/ } \\
\text { Strate } \\
\text { regroupée }\end{array}$ & $\begin{array}{c}\text { Nombre } \\
\text { de } \\
\text { mesures }\end{array}$ & $\begin{array}{l}\text { Nombre total } \\
\text { de tiges } \\
\text { marchandes } \\
\text { (tiges/ha) }\end{array}$ & $\begin{array}{c}\text { Volume } \\
\text { marchand brut } \\
\left(\mathrm{m}^{3} / \mathrm{ha}\right)\end{array}$ & $\begin{array}{c}\text { Diamètre } \\
\text { moyen } \\
\text { quadratique } \\
(\mathrm{cm})\end{array}$ & Volume marchand brut $\left(\mathrm{m}^{3} / \mathrm{ha}\right)$ \\
\hline
\end{tabular}

Nord-Ouest

Fx-MS2-Je-Fa

Ep-RE2-Je-Fa

Ep-RE2-Je-Fo

Ep-RE2-Ma-Fa

Ep-RE2-Ma-Fo

Ep-RE3-Ma-Fa

Ep-RE3-Ma-Fo

Ep-RS2-Je-Fa

Ep-RS2-Ma-Fa

Nord-Est

Ep-RS2-Je-Fa

Ep-RS2-Je-Fo

Ep-RS2-Ma-Fa

Ep-RS2-Ma-Fo

Sb-RS2-Je-Fo

Sb-RS2-Ma-Fa

\section{Ouest}

Fi-MS2-Je-Fo

$\mathrm{Fi}-\mathrm{MS} 2-\mathrm{Ma}-\mathrm{Fa}$

Ep-RE2-Je-Fa

Ep-RE2-Je-Fo

Ep-RE3-Je-Fa

Ep-RS2-Je-Fa

Ep-RS2-Je-Fo

Pg-RE2-Je-Fo

Centre

Fi-MS1-Je-Fa

Fi-MS1-Je-Fo

Fi-MS2-Je-Fo

Fi-RS2-Je-Fo

Ep-RS2-Je-Fo

Sb-MS2-Je-Fa

Sb-MS2-Je-Fo

$\mathrm{Sb}-\mathrm{MS} 2-\mathrm{Ma}-\mathrm{Fa}$

Sb-RS2-Je-Fo

Est

Bb-MS2-Je-Fo

Sb-MS2-Je-Fa

Sb-MS2-Je-Fo

Sb-MS2-Ma-Fa

Sb-MS2-Ma-Fo

Sud-Ouest

Fi-MJ1-Je-Fo

Fi-MJ2-Je-Fa

Fi-MJ2-Je-Fo

$\mathrm{Fi}-\mathrm{MJ} 2-\mathrm{Ma}-\mathrm{Fa}$

Fi-MS2-Je-Fo

Ep-RS2-Je-Fa

Ep-RS2-Je-Fo

$732(525-1075)$
$1029(125-2575)$
$1259(175-2575)$
$944(275-2750)$
$1184(625-1800)$
$827(275-1600)$
$1309(325-2225)$
$717(275-1850)$
$813(325-1275)$

$150(44-335)$

$68(7-148)$

$118(5-410)$

$112(19-241)$

$110(35-345)$

$46(14-172)$

$106(18-191)$

74 (12-188)

$112(17-242)$

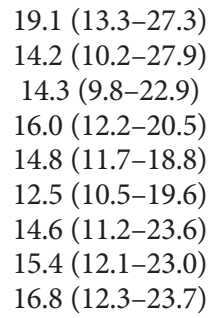

$62(15-127)$

89 (28-238)

$103(19-211)$

$146(42-301)$

$124(21-280)$

171 (81-327)

$136(14-316)$

$134(60-249)$

$53(12-121)$

$90(16-318)$

47 (7-81) 12.4

$87(21-167)$

100 (13-207)

$101(3-228)$

99 (19-211)

118 (14-262)

$76(14-173)$

$102(19-143)$

$112(8-221)$

63 (4-146)

$98(11-195)$

$160(29-280)$

$114(51-243)$

$13.7(10.6-20.1)$
$13.1(11.1-20.4)$
$16.5(12.3-25.6)$
$16.3(11.8-20.1)$
$15.4(10.6-25.2)$
$17.5(13.0-25.3)$

$16.5(10.3-25.3)$

$21.5(14.0-26.1)$

$12.6(10.4-16.6)$

$13.1(10.8-17.4)$

(10.7-14.8)

$15.3(11.4-22.8)$

$13.8(10.8-17.3)$

$12.6(10.7-16.7)$

17.5 (11.4-24.0)

$17.2(11.6-23.2)$

$14.6(11.3-18.8)$

$15.0(11.4-19.5)$

$14.5(10.8-20.2)$

13.8 (11.1-16.1)

$14.1(10.6-26.1)$

$19.3(14.0-26.8)$

$15.1(12.2-21.1)$

97 (19-219)

$133(39-242)$

$150(17-322)$

$216(87-322)$

$171(38-291)$

$14.3(11.3-18.9)$

$15.0(11.5-25.8)$

$14.9(10.2-23.8)$

$20.5(16.8-24.0)$

$19.5(14.0-28.1)$

$126(19-255)$

$83(8-205)$

127 (30-285)

$112(65-255)$

$149(39-250)$

77 (29-161)

86 (19-159)
$18.9(13.5-28.4)$

$19.8(12.6-31.5)$

$18.8(13.2-26.9)$

$20.2(15.7-33.9)$

$17.9(13.0-23.3)$

$14.9(12.2-18.4)$

15.1 (11.9-25.4)
$899(325-1925$

998 (125-1750)

$15.1(11.9-25.4)$

\begin{tabular}{ccccc}
$\mathbf{F i}$ & $\mathbf{F t}$ & $\mathbf{R i}$ & $\mathbf{R t}$ & $\mathbf{S b}$ \\
\hline 90 & 0 & 21 & 29 & 10 \\
0 & 0 & 8 & 59 & 0 \\
20 & 0 & 18 & 76 & 5 \\
0 & 0 & 5 & 105 & 2 \\
0 & 0 & 0 & 109 & 1 \\
0 & 0 & 1 & 43 & 2 \\
0 & 0 & 0 & 105 & 1 \\
5 & 0 & 3 & 65 & 1 \\
10 & 0 & 15 & 81 & 5
\end{tabular}

$\begin{array}{ccccc}1 & 0 & 0 & 38 & 23 \\ 6 & 0 & 3 & 61 & 19 \\ 3 & 0 & 0 & 72 & 28 \\ 6 & 0 & 0 & 92 & 48 \\ 4 & 0 & 0 & 38 & 81 \\ 9 & 0 & 0 & 63 & 100\end{array}$

$\begin{array}{ccccc}102 & 0 & 4 & 10 & 20 \\ 107 & 0 & 5 & 5 & 16 \\ 0 & 0 & 14 & 38 & 1 \\ 3 & 0 & 30 & 56 & 1 \\ 0 & 0 & 3 & 41 & 1 \\ 10 & 0 & 22 & 47 & 8 \\ 10 & 0 & 21 & 63 & 6 \\ 2 & 0 & 60 & 38 & 0\end{array}$

$\begin{array}{ccccc}29 & 21 & 4 & 22 & 23 \\ 72 & 6 & 0 & 25 & 15 \\ 47 & 1 & 0 & 17 & 12 \\ 36 & 1 & 0 & 48 & 17 \\ 14 & 0 & 2 & 83 & 14 \\ 8 & 0 & 0 & 19 & 36 \\ 11 & 0 & 0 & 17 & 70 \\ 24 & 0 & 0 & 45 & 92 \\ 18 & 8 & 0 & 37 & 51\end{array}$

$\begin{array}{ccccc}42 & 0 & 0 & 17 & 37 \\ 10 & 0 & 0 & 34 & 88 \\ 7 & 0 & 0 & 34 & 109 \\ 12 & 0 & 0 & 66 & 138 \\ 1 & 0 & 0 & 59 & 112\end{array}$

27

$\begin{array}{ccccc}58 & 14 & 0 & 23 & 31 \\ 36 & 10 & 1 & 17 & 20 \\ 76 & 13 & 2 & 19 & 17 \\ 54 & 33 & 0 & 15 & 9 \\ 103 & 2 & 1 & 19 & 24 \\ 5 & 0 & 1 & 61 & 11 \\ 13 & 0 & 5 & 60 & 9\end{array}$


lation est de 10 ans, mais que le nombre d'années entre deux mesures de PEP est variable (de 10 à 16 ans), nous avons ajusté les prévisions au nombre d'années entre deux mesures consécutives à l'aide d'une interpolation linéaire entre les prévisions de 2 périodes de simulation consécutives.

Nous avons utilisé les prévisions appliquant le modificateur pour épidémie de TBE lorsquau moins quatre années consécutives de défoliations graves par la TBE étaient observées dans la période séparant la mesure de départ de la simulation et la période d’observation de référence de la PEP. Si moins de quatre années consécutives de défoliations graves étaient observées, les résultats de la simulation sans modificateur étaient utilisés afin dévaluer le comportement des modèles.

Nous avons comparé le biais (Eq. 2) et la racine carrée de lerreur quadratique moyenne (REQM : Eq. 3) des prévisions du volume marchand brut des deux modèles pour chacune des strates regroupées dans chaque territoire :

$$
\begin{aligned}
& \text { [2] biais }=\frac{1}{n} \sum_{i=1}^{n}\left(y_{i}-\hat{y}_{i}\right) \\
& \text { [3] REQM }=\sqrt{\frac{1}{n} \sum_{i=1}^{n}\left(y_{i}-\hat{y}_{i}\right)^{2}}
\end{aligned}
$$

où $y_{i}$ est la valeur observée d'une placette $i, \hat{y}_{i}$ est son estimation et $n$ est le nombre dobservations.

Pour chacune des strates regroupées, nous avons comparé les prévisions moyennes du volume marchand brut, du diamètre moyen quadratique et du nombre de tiges, toutes essences confondues, de chacun des modèles sur une période de simulation de 50 ans. Nous avons utilisé des seuils de $25 \%$ (ou $25 \mathrm{~m}^{3} \cdot \mathrm{ha}^{-1}$ ) pour le volume, de $25 \%$ (ou 250 tiges $\cdot \mathrm{ha}^{-1}$ ) pour la densité et de $15 \%$ pour le diamètre moyen quadratique pour déceler des différences notables de chaque variable. Nous avons aussi comparé la prévision moyenne à 50 ans obtenue avec Artémis à celle obtenue avec Natura à l'aide d'un test de Student pour données appariées, en utilisant le seuil $\alpha=0.05$ pour détecter une différence significative.

De plus, les prévisions moyennes sur 50 ans ont également été comparées aux observations des placettes-échantillons temporaires (PET) des $3^{\mathrm{e}}$ et $4^{\mathrm{e}}$ inventaires forestiers décennaux, afin de nous assurer que les prévisions à long terme des modèles demeuraient dans les limites des valeurs observées pour les mêmes types de peuplements. Pour ce faire, pour chaque strate regroupée, nous avons sélectionné les PET situées sur le même territoire de simulation qui partageaient le groupe d'essences et la végétation potentielle avec la strate regroupée. Nous avons ensuite comparé ces prévisions aux $5^{\mathrm{e}}$ et $95^{\mathrm{e}}$ percentiles des distributions du volume marchand brut, de la densité et du diamètre moyen quadratique pour chaque groupe de PET.

De plus, lorsqu'un effet de la TBE était simulé à la première période de croissance et que la strate regroupée avait un volume marchand brut de sapin baumier d'au moins $25 \mathrm{~m}^{3} \cdot \mathrm{ha}^{-1}$, nous avons comparé les prévisions à long terme du volume marchand brut et du nombre de tiges de sapin baumier de chaque modèle par strates regroupées.

\section{Résultats}

Évaluation des biais et de la racine carrée de l'erreur quadratique moyenne des prévisions de volume marchand brut

Pour les deux modèles, les biais des prévisions sur une période de 10 à 16 ans sont très variables entre les différentes strates et entre les différents territoires à létude (Fig. 2). De manière générale, les prévisions obtenues avec Artémis sont moins biaisées que celles obtenues avec Natura dans les territoires Ouest, Est et Sud-Ouest, alors que les prévisions obtenues avec Natura sont moins biaisées dans les territoires Nord-Ouest, Nord-Est, et Centre (Tableau 4). Natura a produit des estimations moins biaisées qu'Artémis pour les pessières matures et les sapinières, à l'exception des sapinières matures sur la végétation potentielle MS2 (Fig. 2). La densité des strates a eu peu d'effet sur le biais des prévisions (Fig. 2).

La REQM des modèles varie aussi entre les territoires (Tableau 4). Mis à part le territoire Centre, où Artémis est beaucoup moins précis que Natura, les différences entre les modèles sont moins marquées pour la REQM que pour le biais (Tableau 4). Parmi les différentes strates, les deux modèles ont produit la REQM la plus faible pour les strates dépinettes noires (Picea mariana [Mill.] B.S.P.) sur la végétation potentielle RE3 du territoire Ouest $\left(13,3\right.$ et $11,6 \mathrm{~m}^{3} \cdot \mathrm{ha}^{-1}$ pour Artémis et Natura, respectivement), alors que l'erreur la plus élevée est produite pour les strates de sapins matures sur la végétation potentielle MS2 du territoire Est (71,0 et $82,3 \mathrm{~m}^{3} \cdot \mathrm{ha}^{-1}$ pour Artémis et Natura, respectivement, Fig. 3).

\section{Comparaison des prévisions sur $\mathbf{5 0}$ ans}

Le comportement des deux modèles est souvent semblable, mais varie beaucoup d'un territoire et d'une strate à l'autre ${ }^{3}$. Les deux modèles prévoient généralement une augmentation $\mathrm{du}$ volume pour les strates jeunes et une stagnation ou une diminution de volume pour les strates plus âgées (Fig. 4). Ce patron dévolution est plus marqué pour Natura, avec lequel 11 strates matures sur 14 montrent une stagnation ou une diminution de volume marchand brut dès le début de la simulation.

Les volumes prévus par Artémis et Natura se situent généralement entre le $5^{\mathrm{e}}$ et le $95^{\mathrm{e}}$ percentile de la distribution du volume marchand brut des PET. Parfois, cependant, les modèles prévoient des volumes exceptionnellement hauts ou exceptionnellement bas. Artémis prévoit des volumes plus importants que le $95^{\mathrm{e}}$ percentile pour une seule strate, soit celle des épinettes noires jeunes sur la végétation potentielle RS2 avec une forte densité, sur le territoire SudOuest. Les prévisions de Natura dépassent le $95^{\mathrm{e}}$ percentile dans neuf strates situées sur les territoires Centre et Est, desquelles six sont composées de sapinières sur la végétation potentielle MS2.

Pour 26 des 44 strates, le test de Student pour données appariées sur les volumes prédits par Artémis et Natura a révélé une différence significative à 50 ans. Par contre, pour huit de ces strates, la différence de volume est inférieure à $25 \mathrm{~m}^{3} \cdot \mathrm{ha}^{-1}$ ou à $25 \%$ du volume marchand brut. Le territoire

\footnotetext{
${ }^{3}$ S1-Résultats de simulation, toutes essences confondues, du volume marchand brut, du diamètre moyen quadratique et du nombre de tiges à l'hectare sur 50 ans, pour toutes les strates. Matériel supplémentaire disponible en ligne.
} 

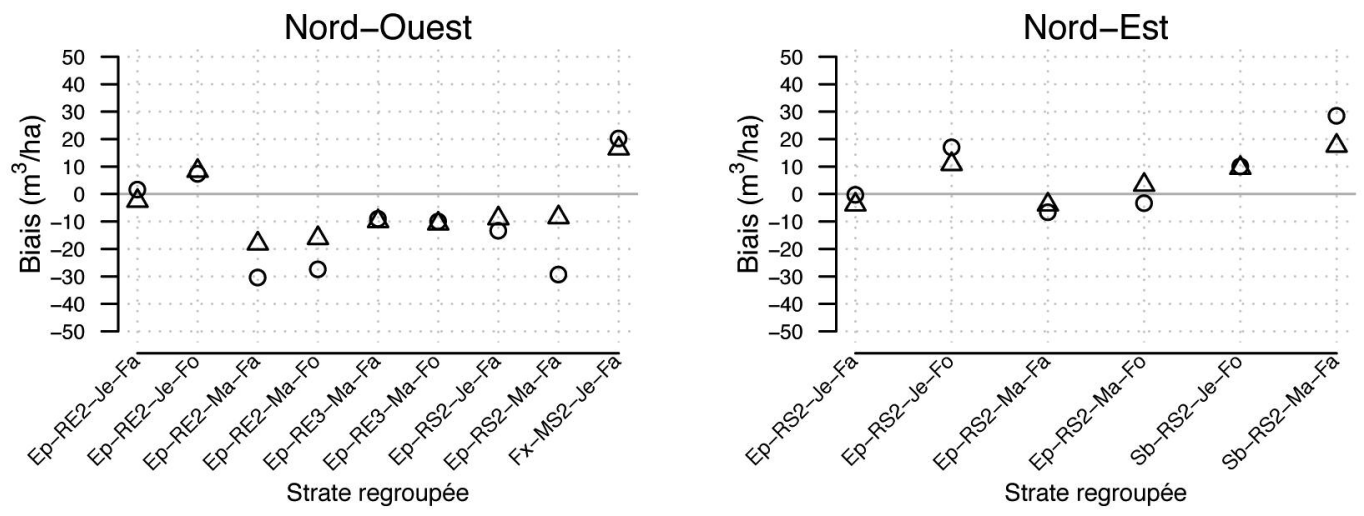

Ouest
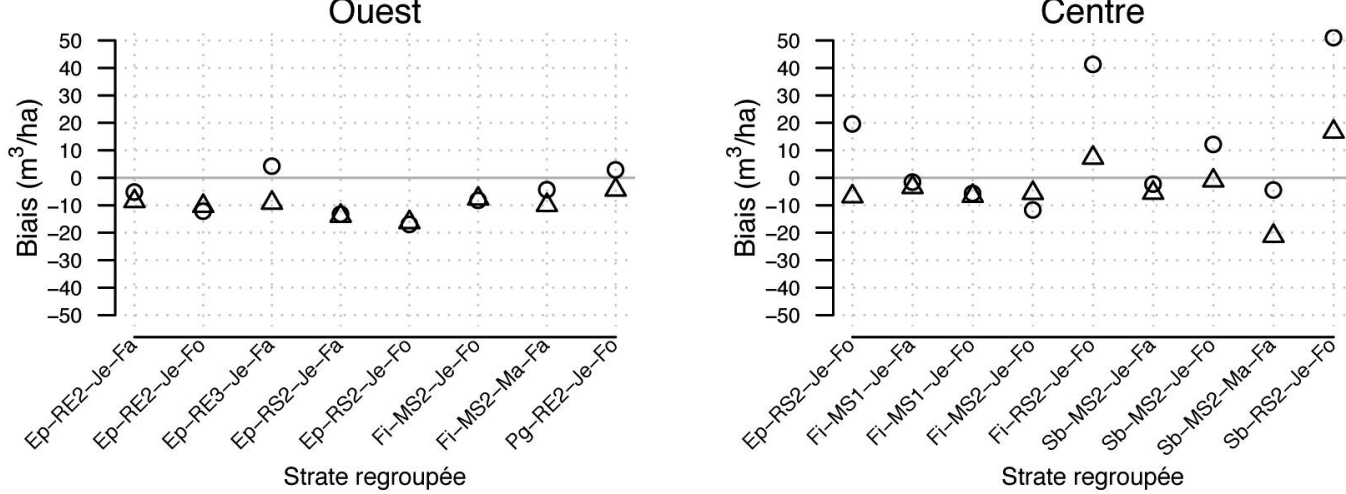

Est
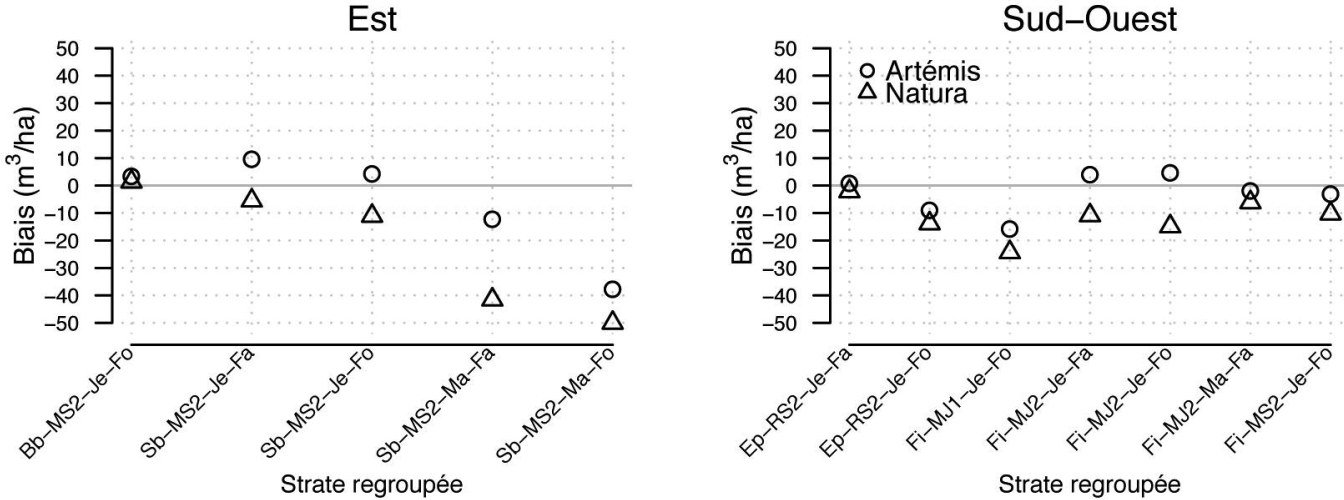

Fig. 2. Biais des prévisions du volume marchand brut, toutes essences confondues, sur une période moyenne de 10 ans, par territoire de simulation et en fonction des strates regroupées

Nord-Ouest pour les strates d'épinettes matures (à l'exception de celles sur la végétation potentielle RE3), le territoire Centre pour les strates de sapin et de feuillus intolérants à forte densité, et le territoire Est pour les strates de sapin sont les endroits où l'on observe les plus grandes différences entre les modèles (Fig. 5). Pour le territoire Nord-Ouest, Artémis prévoit de plus grands volumes que Natura, alors que pour les sapinières et les peuplements de feuillus intolérants des territoires Centre et Est, Natura prévoit les plus grands volumes.
Le diamètre moyen quadratique des arbres varie peu entre les modèles durant les 50 années de simulation. Pendant la période de simulation, la différence entre les estimations des modèles n'a jamais excédé $15 \%$ de la valeur du diamètre moyen quadratique; les valeurs varient de 10 à $20 \mathrm{~cm}$, sauf dans le territoire Sud-Ouest. Sur celui-ci, les deux modèles prévoient, pour les strates de feuillus intolérants, des diamètres moyens quadratiques qui atteignent de 20 à $25 \mathrm{~cm}$ après 50 ans de simulation. Lorsque lon compare les prévi- 
Tableau 4. Biais et racine carrée de l'erreur quadratique moyenne (REQM) des prévisions des modèles Artémis et Natura pour le volume marchand brut, toutes essences confondues et pour les 6 territoires étudiés, sur une période moyenne de 10 ans.

\begin{tabular}{llcccc}
\hline Modèle & Territoire & $\begin{array}{c}\text { Biais } \\
\left(\mathbf{m}^{\mathbf{3}} \mathbf{h a} \mathbf{h}\right)\end{array}$ & $\begin{array}{c}\text { Biais } \\
\text { relatif } \\
\mathbf{( \% )}\end{array}$ & $\begin{array}{c}\text { REQM } \\
\left(\mathbf{m}^{\mathbf{3}} / \mathbf{h a}\right)\end{array}$ & $\begin{array}{c}\text { REQM } \\
\text { relative } \\
(\mathbf{\%})\end{array}$ \\
\hline Artémis & & & & & \\
& Nord-Ouest & -10.0 & -14.0 & 34.5 & 37.4 \\
& Nord-Est & 7.6 & 4.6 & 34.7 & 27.1 \\
& Ouest & -6.6 & -5.8 & 22.6 & 22.3 \\
& Centre & 11.0 & 7.5 & 40.1 & 32.0 \\
& Est & -6.5 & -4.9 & 44.4 & 30.3 \\
& Sud-Ouest & -1.8 & -1.8 & 31.1 & 25.2 \\
& Moyenne Artémis & $-\mathbf{1 . 0}$ & $-\mathbf{2 . 5}$ & $\mathbf{3 3 . 9}$ & $\mathbf{2 9 . 0}$ \\
& & & & & \\
Natura & & & & \\
& Nord-Ouest & -5.5 & -9.2 & 29.0 & 32.0 \\
& Nord-Est & 5.6 & 3.2 & 31.3 & 24.8 \\
& Ouest & -10.0 & -10.5 & 20.8 & 20.5 \\
& Centre & -2.9 & -2.7 & 31.8 & 26.1 \\
& Est & -22.0 & -15.2 & 49.5 & 34.0 \\
& Sud-Ouest & -12.7 & -10.3 & 32.1 & 25.6 \\
Moyenne Natura & -7.8 & -7.5 & $\mathbf{3 1 . 3}$ & $\mathbf{2 6 . 8}$ \\
\hline
\end{tabular}

sions aux diamètres moyens quadratiques observés dans les PET, les diamètres prévus par Artémis se situent tous entre le $5^{\mathrm{e}}$ et le $95^{\mathrm{e}}$ percentile de la distribution. Ceux prévus par Natura sont plus grands que le $95^{\mathrm{e}}$ percentile pour deux strates de sapins situées dans les territoires Centre et Est.

Pour Artémis, la densité de tiges marchandes prévues après 50 ans de simulation varie de 420 tiges $\mathrm{ha}^{-1}$ (pour une strate d'épinettes noires matures de faible densité du territoire Sud-Ouest) à 1210 tiges $\mathrm{ha}^{-1}$ (pour une strate d'épinettes noires jeunes de forte densité du territoire Nord-Est). Pour Natura, le nombre prévu de tiges marchandes après 50 ans de simulation varie de 460 tiges.ha ${ }^{-1}$ (pour une strate dépinettes noires matures de faible densité du territoire Sud-Ouest) à 1790 tiges $\cdot \mathrm{ha}^{-1}$ (pour une strate de jeunes sapins baumiers du territoire Centre). Les deux modèles prévoient, pour lensemble des strates, des densités comparables à celles retrouvées dans les PET qui partagent les mêmes caractéristiques. Pour 24 des 44 strates à létude, le test de Student pour données appariées a révélé des différences significatives entre les densités prévues par les deux modèles. Cependant, pour 11 des 24 strates, la différence entre les prévisions des deux modèles était inférieure à $25 \%$ ou 250 tiges ha $^{-1}$. Les 13 strates présentant les plus grandes différences sont situées dans les territoires Nord-Est, Centre, Ouest et Est. Tous ces cas surviennent pour des strates jeunes, dans lesquelles Natura prévoit plus de tiges qu’Artémis.

\section{Comparaison des prévisions sur 50 ans avec défoliation par la TBE à la première période}

Dans les territoires à létude, autant pour le nombre de tiges que pour le volume de sapin baumier (strates avec au moins $25 \mathrm{~m}^{3} \cdot \mathrm{ha}^{-1}$ de sapin baumier au début de la simulation), la courbe dévolution avec un effet de la TBE à la première période prévoit toujours une baisse du volume de sapin baumier à la première période de simulation ${ }^{4}$. La baisse est plus marquée dans les peuplements plus âgés que dans les peuplements plus jeunes, et ce, pour les deux modèles (Fig. 6). Sauf dans les strates du territoire Nord-Est, cette baisse est généralement suivie d'une augmentation plus ou moins marquée du volume de sapin baumier pour les périodes suivantes. De manière générale, leffet de la défoliation par la TBE est plus marqué avec Artémis qu’avec Natura. Lors de la première période de simulation avec un effet de la TBE, Artémis prévoit une diminution du volume marchand de sapin variant de $72 \%$ (territoire Est, strate Sb-MS2-Ma-Fa) à 17 \% (territoire Sud-Ouest, strate Fi-MJ1-Je-Fo). Pour le même modèle et la même période la densité de sapin diminue dans les mêmes strates de 66 et de $21 \%$, respectivement. Avec Natura, pour la première période de simulation avec défoliation par la TBE, la réduction du volume prévue pour le sapin varie de $53 \%$ (territoire Nord-Est, strate Ep-RS2-Ma-Fo) à $10 \%$ (territoire Centre, strate Sb-MS2-Je-Fa), alors que celle prévue pour la densité de sapin diminue de 42 à $14 \%$ pour les mêmes strates.

\section{Discussion}

Évaluation et comparaison des biais des prévisions des modèles De façon générale, le biais sur la prévision du volume marchand brut, toutes essences confondues, varie dans chacun des territoires en fonction de la strate d'aménagement. Pour Natura, il passe d'une sous-estimation de $18 \mathrm{~m}^{3} \cdot \mathrm{ha}^{-1}$ à une surestimation de $53 \mathrm{~m}^{3} \cdot \mathrm{ha}^{-1}$, alors que pour Artémis, il passe d'une sous-estimation de $51 \mathrm{~m}^{3} \cdot \mathrm{ha}^{-1}$ à une surestimation de $38 \mathrm{~m}^{3} \cdot \mathrm{ha}^{-1}$. La REQM, quant à elle, varie de 12 à $84 \mathrm{~m}^{3} \cdot \mathrm{ha}^{-1}$ et de 13 à $72 \mathrm{~m}^{3} \cdot \mathrm{ha}^{-1}$ pour Natura et Artémis, respectivement. Le modèle Natura surestime le volume dans 35 strates et le sous-estime dans neuf strates. Artémis, quant à lui, surestime le volume dans 26 strates et le sous-estime dans 18 strates. Nous n'avons observé aucun patron général de biais entre les strates de même essence, même végétation potentielle, même âge ou même densité dans les différents territoires. Cependant, pour Natura, les biais les plus forts semblent présents dans les sapinières matures, notamment celles du territoire Est sur la végétation potentielle MS2. Dans ces peuplements matures, le biais important de Natura (et d'Artémis pour la strate de densité la plus forte) peut être attribué à la perte importante de volume survenue dans quelques placettes, pertes qui pourraient être dues à des défoliations d'insectes non captées par les modèles et à la présence de chablis partiels liés à la sénescence. En ce qui concerne Artémis, les biais les plus forts sobservent dans différentes strates de sapins, d'épinettes et de feuillus intolérants.

Lidentification des placettes ayant subi une défoliation par la tordeuse des bourgeons de l'épinette avait déjà été soulignée dans Power (2016) et Auger (2017a) comme une cause de biais, compte tenu de l'imprécision liée à cette information. Des efforts devront être consentis afin de mieux identifier les

${ }^{4}$ S2-Résultats de simulation du volume marchand brut, du diamètre moyen quadratique et du nombre de tiges à l'hectare de sapin baumier sur 50 ans avec ou sans défoliation par la tordeuse des bourgeons de lépinette. Matériel supplémentaire disponible en ligne. 


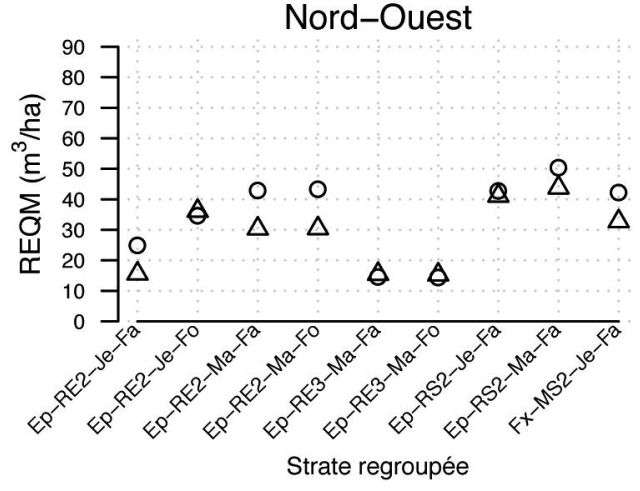

Ouest

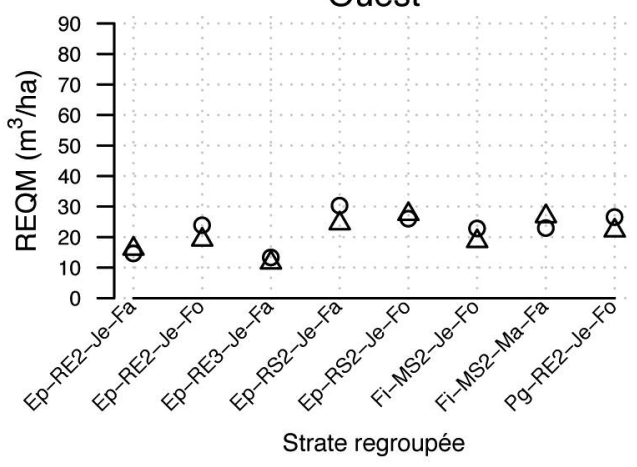

Est

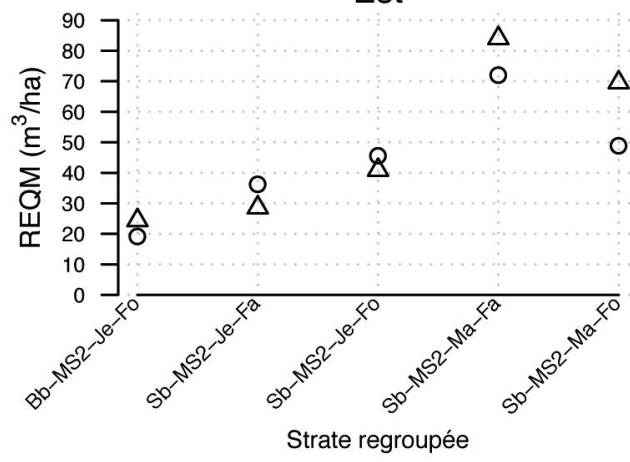

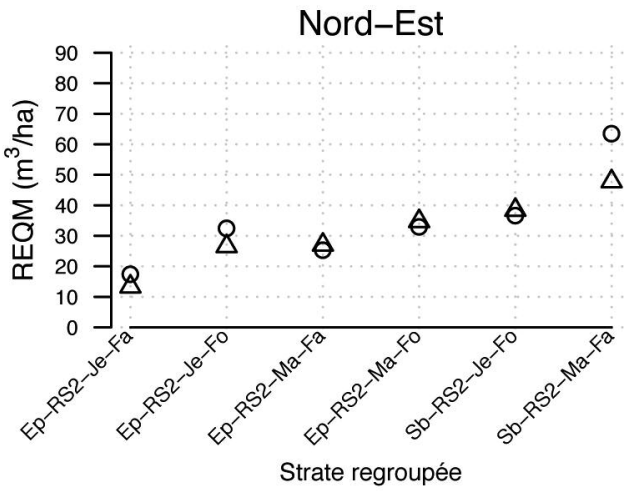

Centre

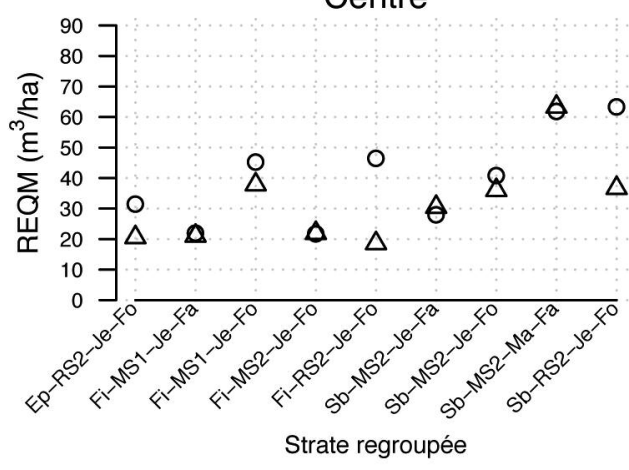

Sud-Ouest

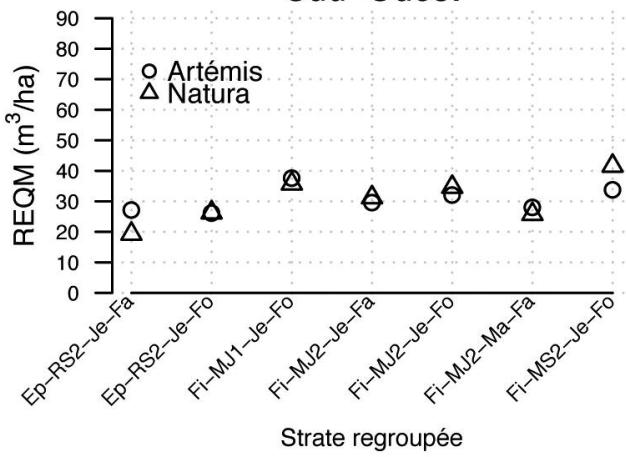

Fig. 3. Racine carrée de l'erreur quadratique moyenne (REQM) des prévisions du volume marchand brut, toutes essences confondues, sur une période moyenne de 10 ans, par territoire de simulation et en fonction des strates regroupées

peuplements affectés par des défoliations graves, et ce, afin d’améliorer le comportement des prochaines versions des modèles.

Les deux strates de sapins matures du territoire Est sont parmi celles qui comptent le plus petit nombre de mesures. Ces résultats viennent donc aussi souligner la variabilité de la qualité des prévisions des modèles, notamment pour les petits groupes d'observations. Les utilisateurs des modèles devraient porter une attention particulière à ce point.

Les territoires du nord, où Natura performe mieux qu'Artémis, sont situés dans le domaine bioclimatique de la pessière à mousses, une région où les peuplements d'épinettes noires couvrent la vaste majorité du territoire (MRN 2013) et où ceux-ci, particulièrement dans l'ouest de la province, sont souvent de structure régulière (Boucher et al. 2003). Il est donc probable que pour ce type de peuplements, un modèle à léchelle du peuplement comme Natura, qui modélise directement le changement en volume marchand brut, soit plus approprié qu'Artémis, qui estime l'accroissement en volume à partir de la sommation de laccroissement de chacun des arbres pris individuellement. Des résultats semblables ont été obtenus par McCullagh et al. (2017) et Mäkinen et al. (2008), 


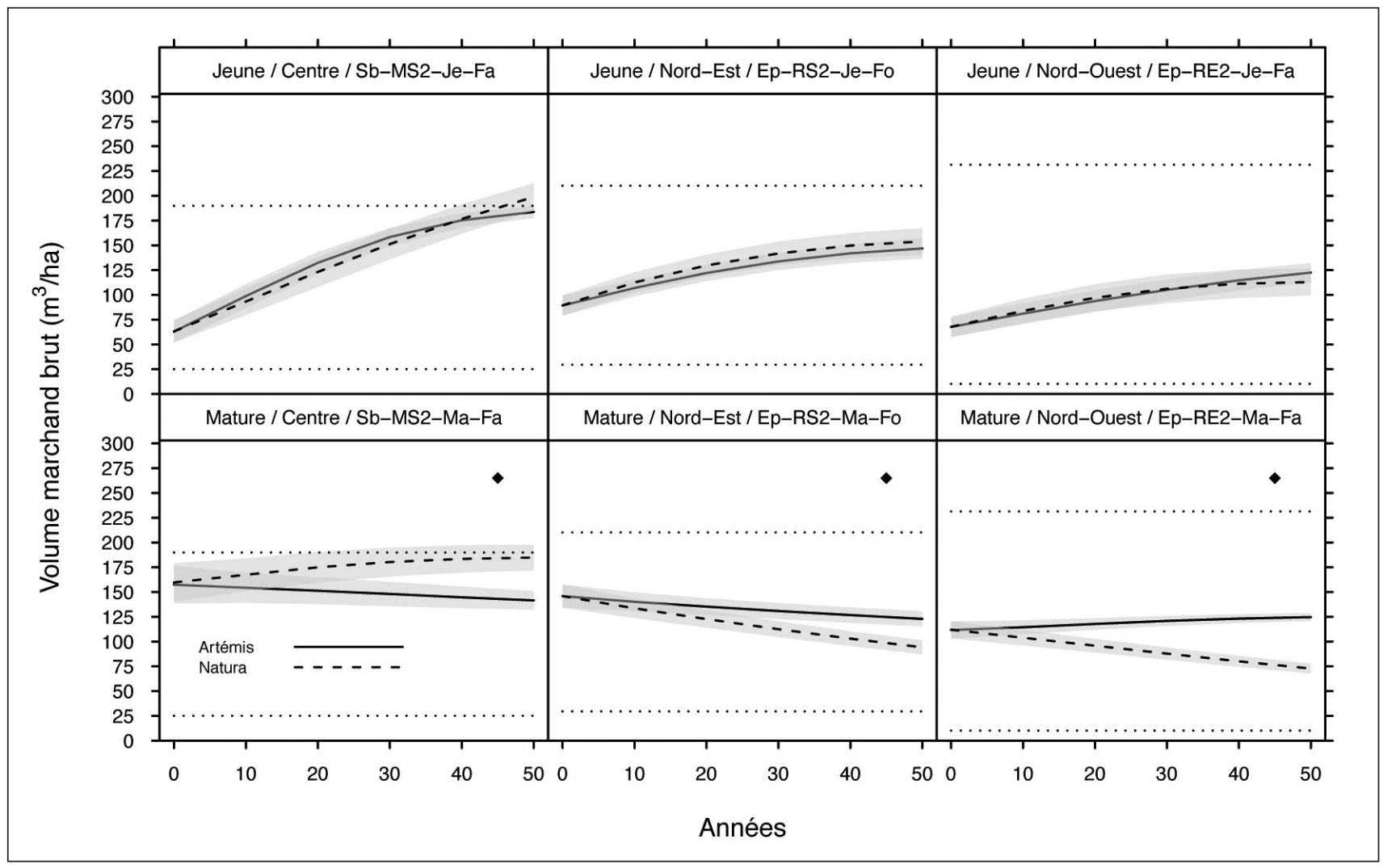

Fig. 4. Évolution moyenne sur 50 ans selon le modèle de croissance des prévisions du volume marchand brut, toutes essences confondues, pour des strates regroupées de classe d'âge jeune et mature. Les lignes horizontales pointillées représentent les $5^{\mathrm{e}}$ et $95^{\mathrm{e}}$ percentiles de la distribution des valeurs observées dans les placettes-échantillons temporaires. Le losange noir indique une différence significative entre les prévisions des modèles à 50 ans au seuil $\alpha=0.05$. Les bandes grisées délimitent l'intervalle de \pm 1 erreur type de la prévision moyenne.

qui rapportent que dans des peuplements monospécifiques de structure équienne, un modèle à l'échelle du peuplement performait mieux qu'un modèle à l'échelle de l'arbre.

Les valeurs de biais et de REQM observées sur le volume, pour les deux modèles, sont comparables à celles publiées par Härkonen et al. (2010), qui ont observé des biais variant de 0 à $5 \%$ et des REQM variant de 25 à $30 \%$ sur le volume sur une période de simulation de 11 ans. Leur territoire détude couvrait tout le sud de la Finlande et comptait 837 placetteséchantillons. Les valeurs de biais et de REQM que nous avons observées sur le volume sont également comparables à celles obtenues par McCullagh et al. (2017), lorsqu'ils ont comparé les résultats obtenus par un modèle à léchelle de l’arbre et un modèle à l'échelle du peuplement pour une simulation de cinq ans de 200 placettes dépinette de Sitka (Picea sitchensis [Bong.] Carr.) en Irlande.

Il est intéressant de remarquer que le modèle Artémis réussit à bien estimer le volume total moyen des strates, étant donné qu'il fonctionne à léchelle de l'arbre et que la variation $\mathrm{du}$ volume du peuplement correspond à la variation du volume de chacune des tiges prises individuellement. Natura, quant à lui, modélise l'évolution du volume marchand à léchelle du groupe dessences, ce qui constitue un niveau supérieur d'agrégation. Par contre, Natura performe mieux qu'Artémis dans 27 des 44 strates selon les biais des prévisions du nombre de tiges, de même que dans 32 strates sur 44 selon ceux du diamètre moyen quadratique ${ }^{5}$. Ce résultat s'explique probablement par le fait que Natura simule directement le nombre de tiges et la surface terrière (deux intrants du calcul du diamètre moyen quadratique) alors que pour Artémis, ces valeurs sont dérivées des caractéristiques de chacun des arbres du peuplement. Il est également intéressant de noter que les territoires où Artémis produit de façon générale de meilleures prévisions pour le nombre de tiges et le diamètre moyen quadratique sont ceux de l'Est et du Sud-Ouest. Ces résultats pourraient indiquer que la simulation de la croissance de ces forêts, généralement moins homogènes en composition, se fait mieux à l'aide de modèles à léchelle de l'arbre, et que les modèles à léchelle du peuplement représentent plus difficilement les différences de croissance entre les arbres de différentes espèces et de différentes tailles (Porté et Bartelink 2002).

Les différences de biais en volume entre les strates montrent que la performance des modèles Artémis et Natura est extrêmement variable d'un territoire à l'autre et d'une strate regroupée à l'autre. Une telle variation de la performance d'un modèle en fonction de lemplacement et de la composition du

${ }^{5}$ S3-Biais en en densité et en diamètre moyen quadratique pour l'ensemble des strates et des territoires de simulation. Matériel supplémentaire disponible en ligne. 


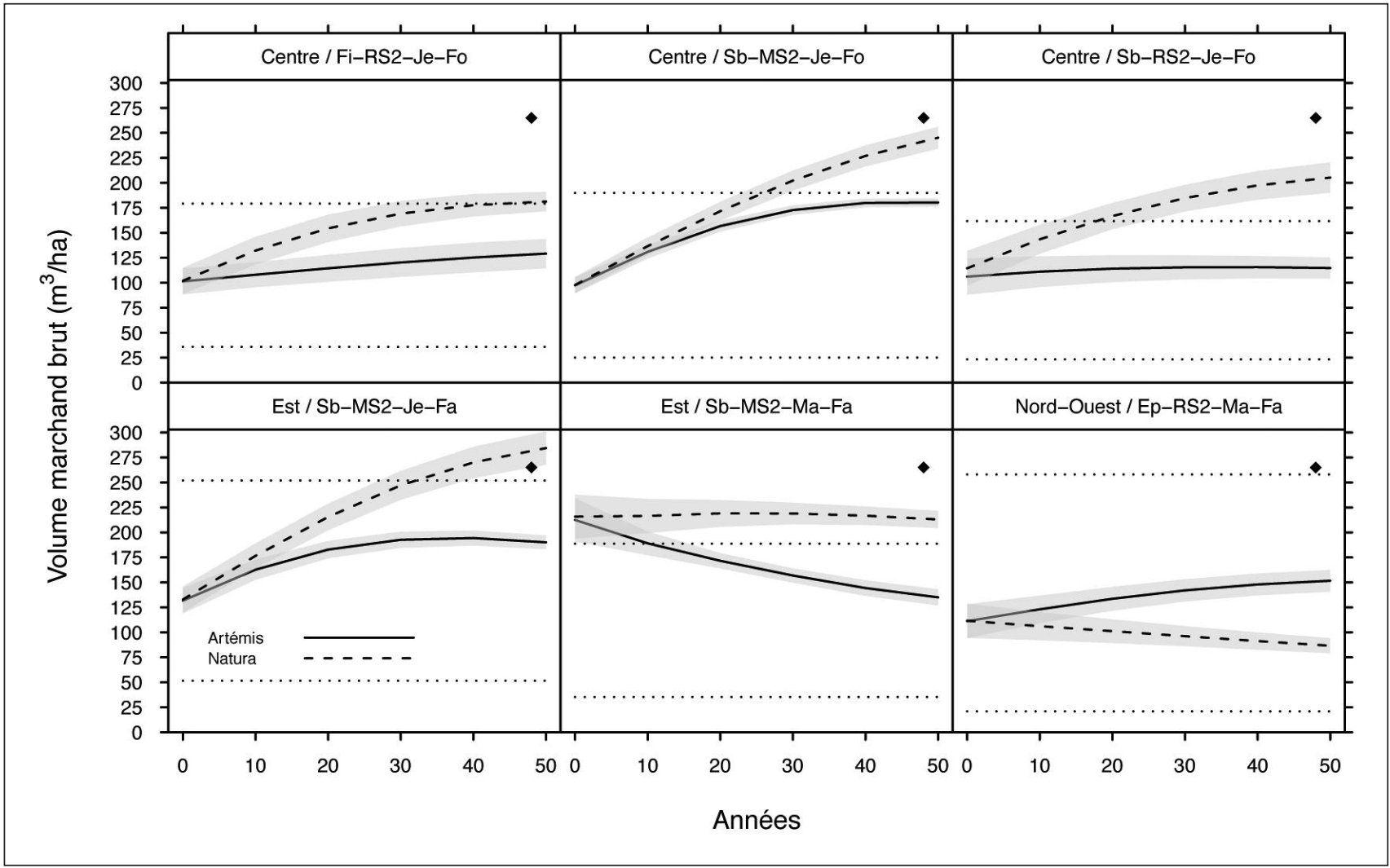

Fig. 5. Évolution moyenne sur 50 ans selon le modèle de croissance des prévisions du volume marchand brut, toutes essences confondues, pour des strates regroupées pour lesquelles les modèles Artémis et Natura n'ont pas le même comportement. Les lignes horizontales pointillées représentent les $5^{\mathrm{e}}$ et $95^{\mathrm{e}}$ percentiles de la distribution des valeurs observées dans les placettes-échantillons temporaires. Le losange noir indique une différence significative entre les prévisions des modèles à 50 ans au seuil $\alpha=0.05$. Les bandes grisées délimitent l'intervalle de \pm 1 erreur type de la prévision moyenne.

peuplement a également été remarquée par Härkonen et al. (2010). Elle provient probablement du fait qu'Artémis et Natura sont des modèles généraux qui s'appliquent à l’ensemble des types forestiers du territoire québécois. Les avantages de tels modèles sont qu'ils peuvent s'appliquer sur un large territoire et qu'ils ont une bonne performance générale. Leur inconvénient est qu'ils peuvent difficilement performer adéquatement sur chacune des portions du territoire et pour chacun des types de peuplements (von Gadow et Hui 2001). Malgré l'inclusion de variables explicatives telles que le sousdomaine bioclimatique, la température et les précipitations annuelles moyennes, il semble quà léchelle locale, il persiste des disparités que les modèles narrivent pas à toutes capter.

\section{Comportement des modèles à long terme}

Pour les deux modèles, le comportement à long terme semble généralement adéquat puisque les prévisions de volume, de densité et de diamètre moyen demeurent, dans la plupart des cas, dans le spectre dobservation des PET partageant les mêmes caractéristiques. Les différences significatives observées pour certaines strates présentant une différence de volume inférieure à $25 \mathrm{~m}^{3} \cdot \mathrm{ha}^{-1}$, soulignent la faible valeur des erreurs types sur le volume moyen de la plupart des strates (Fig. 4 et 5). Ces faibles valeurs sont dues à la faible variabilité intra-strate et au nombre parfois relativement élevé de mesures dans la strate. La faible variabilité intra-strate peut être attribuée à la sélection de placettes partageant les mêmes caractéristiques afin de constituer les strates. Elle confirme la cohérence des résultats de simulation des modèles lorsque ceux-ci sont employés à simuler des placettes dont la composition, la végétation potentielle, lâge et la densité se ressemblent.

Puisque Artémis et Natura ont été en grande partie paramétrés avec les mêmes données, les différences observées entre les deux modèles quant à la trajectoire de leurs courbes peuvent être attribuées à leur structure. Ainsi, le déclin quasi systématique du volume dans les strates matures et l'augmentation du volume dans les strates jeunes peuvent être attribué à l'utilisation par Natura de lâge comme variable explicative, celle-ci étant l'une des variables ayant le plus d'effet sur l'évolution du volume marchand (Auger 2017a). Lâge nétant pas une variable explicative du modèle Artémis (Power 2016), le comportement de ce modèle est plutôt influencé par la végétation potentielle, lessence, la compétition et différentes variables climatiques. Le fait qu'Artémis fasse abstraction de lâge pourrait expliquer sa surestimation du volume dans les pessières matures des territoires Nord-Est et Nord-Ouest. Il est probable que ces peuplements âgés entrent en sénescence, phénomène qui serait mieux capté par Natura que par Artémis. Cette hypothèse est étayée par le fait que les prévisions d'Artémis sont les plus biaisées pour les vieilles pessières de l'Ouest. Ces peuplements étant de structure plus régulière 


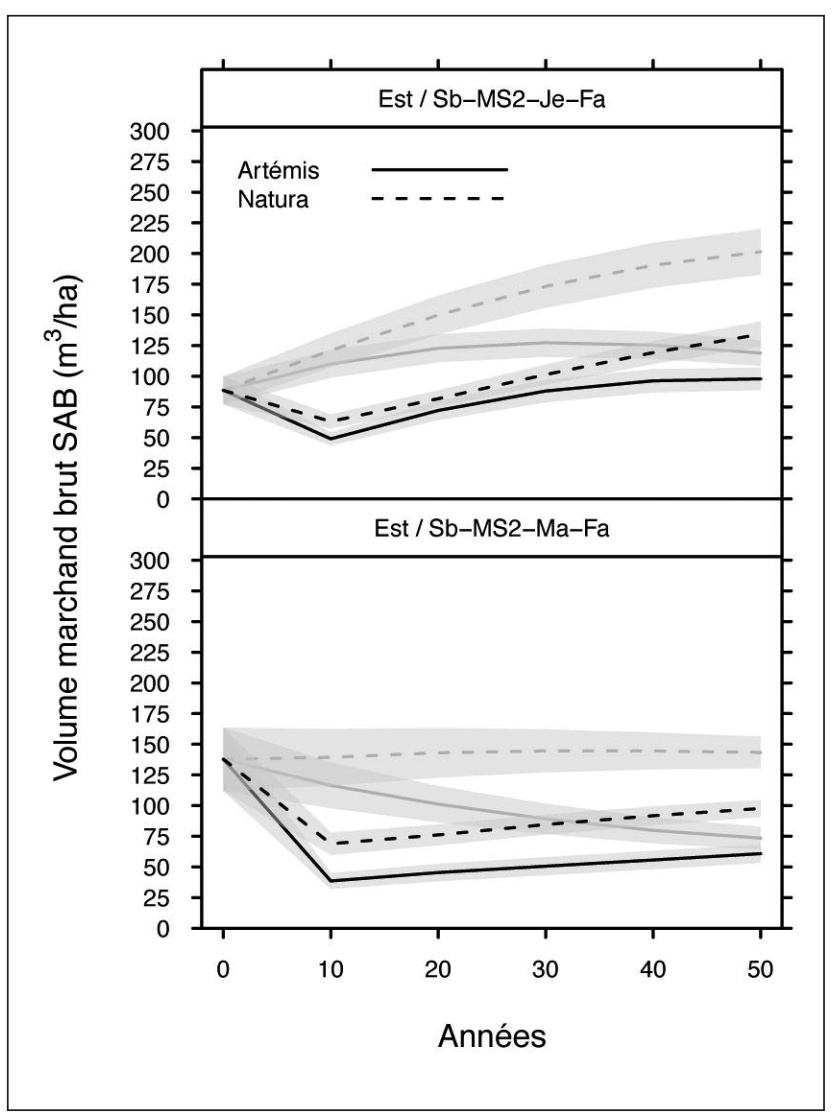

Fig. 6. Évolution moyenne sur 50 ans selon le modèle de croissance des prévisions du volume marchand brut de sabin baumier (SAB) avec (noir) et sans (gris) défoliation causée par la tordeuse des bourgeons de l'épinette durant la première décennie pour 2 strates de sapinière du territoire Est, pour les classes d'âge jeune (graphique du haut] et mature (graphique du bas). Les bandes grisées délimitent l'intervalle de \pm 1 erreur type de la prévision moyenne.

(Boucher et al. 2003), ils pourraient voir leur volume baisser plus rapidement que les pessières irrégulières de l'Est (Garet 2008). Il est toutefois intéressant de constater que, malgré le fait qu'Artémis n'inclue pas lâge comme variable explicative, ce modèle réussit néanmoins à produire des simulations différentes pour des strates dâge variable. Cette capacité du modèle provient du fait que les tiges sont plus petites dans les peuplements jeunes et que, malgré une diminution importante du nombre de tiges, leur accroissement en diamètre permet d’augmenter le volume du peuplement.

\section{Simulation des peuplements défoliés par la tordeuse des bour- geons de l'épinette}

Artémis et Natura sont conçus pour que leurs prévisions tiennent compte de loccurrence de défoliations graves causées par la TBE. L’effet TBE des deux modèles concerne principalement le sapin baumier et, dans une moindre mesure, les épinettes. L’effet TBE d'Artémis augmente la probabilité de mortalité des sapins et des épinettes, diminue leur accroissement diamétral et augmente le recrutement dans les périodes suivant son application. Laugmentation du recrutement et la diminution de la compétition pour les tiges survivantes, de même que le ralentissement de la croissance des strates non touchées par la TBE, pourraient être responsables du fait quà long terme, la courbe de volume des strates affectées se rapproche de celles des strates épargnées par la TBE. Ces résultats sont cohérents avec ceux de MacLean et Andersen (2008), qui ont observé que les différences de volume demeuraient entre les peuplements attaqués à divers degrés par la TBE, même 45 ans après défoliation. La diminution progressive des écarts entre les prévisions des strates défoliées et non défoliées semble cohérente, puisque l'effet de la TBE s'atténue à mesure que de nouvelles tiges viennent remplacer les arbres morts. Il est à noter que l'application de l'effet TBE nélimine jamais tous les sapins dans les strates simulées. Ce comportement est attribuable au fait que cette fonctionnalité des modèles a été paramétrée avec un groupe de peuplements frappés de défoliations modérées à graves. Le degré de défoliation variant à l'intérieur de ce groupe, la mortalité dans les peuplements ayant subi les pires effets de la TBE se trouve compensée par celle des peuplements ayant subi des effets moins graves. Dans ce contexte, les modèles seraient appropriés pour simuler l'effet de la TBE sur un groupe de peuplements subissant des défoliations modérées à graves. Par contre, pour certains peuplements plus gravement attaqués par la TBE, les modèles pourraient sous-estimer la mortalité attribuable à cette perturbation. Il faut également considérer quen présence d'une faible quantité de sapin baumier dans le peuplement $\left(<25 \mathrm{~m}^{3} \cdot \mathrm{ha}^{-1}\right)$, l'effet de la défoliation par la TBE pourrait ne pas être suffisant pour compenser laccroissement en volume de sapin baumier. En conséquence, la simulation de défoliation par la TBE dans ce type de peuplement pourrait mener à des résultats incohérents pour le sapin baumier.

\section{Choix des modèles}

Étant donné que les modèles Artémis et Natura peuvent tous deux être utilisés sur les mêmes territoires, il est légitime de se demander lequel utiliser en quelle circonstance. Â cause de la quantité limitée de PEP dans les territoires à létude, ce sont les mesurages, et non les placettes, que nous avons classés en strates. Ainsi, lévaluation par comparaison des prévisions aux données mesurées a seulement pu être effectuée pour un horizon temporel variant de 10 à 16 ans, compte tenu du faible nombre de mesures pour certaines strates.

La simulation des strates de sapin sur la végétation potentielle MS2 semble problématique avec les deux modèles, mais plus particulièrement avec Natura. En plus d'un fort biais à court terme pour les strates matures, les prévisions de volume de Natura ont tendance à surpasser les valeurs observées dans les PET. Conséquemment, à court terme, Artémis devrait être privilégié pour simuler lévolution de ce type de peuplements.

De manière générale, Artémis tend à moins bien simuler les strates dépinettes que Natura. Malgré cela, des doutes peuvent être émis quant aux prévisions de la croissance dans les strates dépinettes avec Natura sur la végétation potentielle RE3 dans le territoire Ouest. Bien que les biais de la première période de croissance de Natura et Artémis se ressemblent, Natura prévoit, à plus long terme, une augmentation marquée du volume total, principalement à cause d'un accroissement du volume de pin gris. Conséquemment, Artémis devrait être privilégié dans ce cas précis, alors que Natura serait plus indiqué pour les autres strates d'épinettes.

Compte tenu des résultats obtenus, l'utilisateur devrait toujours s'interroger et évaluer de manière critique les prévisions 
d'un modèle. Lorsque les prévisions d'Artémis et de Natura sont cohérentes et diffèrent peu à long terme, l'utilisateur peut accorder une plus grande confiance aux résultats des simulations. Dans le cas contraire, l'utilisateur devrait se questionner sur la validité des prévisions de chacun des modèles. À défaut de pouvoir écarter un modèle, il pourrait procéder à une inférence multimodèle comme celle de la méthode du modèle moyen bayésien (van Oijen et al. 2013) afin de prévoir lévolution des strates et diminuer l'incertitude associée aux prévisions. Ces méthodes permettent dobtenir une seule prévision qui combine les résultats de plusieurs modèles.

La cohérence des prévisions d'Artémis et Natura sur 50 ans dévolution nous amène à avoir confiance dans les prévisions des deux modèles sur cet horizon temporel. En effet, dans la majorité des cas, leurs trajectoires se ressemblent. De plus, l'analyse du sens des biais sur le volume total semble indiquer que la direction de la courbe est adéquate. Par contre, à plus long terme, comme sur l'horizon de 150 ans utilisé pour le calcul des possibilités forestières, il devient très difficile de comparer les modèles puisque nous ne disposons pas de données permettant de valider leur évolution à aussi long terme. Nous croyons donc qu'il est toujours judicieux, comme le mentionnent Fortin et Langevin (2010) ainsi que Pothier et Auger (2011), de limiter l'utilisation d'Artémis et Natura à des périodes dévolution ne dépassant pas 60 ans. Cette recommandation s'appuie également sur les constats de Fortin et Langevin (2010) qui mentionnaient que lerreur associée aux prévisions d'Artémis augmentait rapidement dans le temps.

\section{Conclusion}

Lévaluation des modèles de croissance est un processus continu qui fait partie intégrante du processus de modélisation. Les résultats de cette étude montrent que les modèles Artémis et Natura produisent généralement des prévisions cohérentes à l'échelle de la strate. Cependant, l'exactitude de ces prévisions varie grandement d'un territoire et d'un groupe de peuplements à lautre. Même si Artémis produit ses prévisions à l'échelle de l'arbre, il réussit à produire des prévisions à l'échelle de la strate d'une exactitude souvent comparable à celle de Natura, un modèle à léchelle du peuplement. Puisque les modèles Artémis et Natura sont disponibles pour effectuer des simulations pour lensemble du territoire forestier du Québec, l'utilisateur devrait toujours se questionner sur le choix du modèle à adopter et analyser de façon critique les prévisions du modèle choisi. Nous encourageons l'utilisateur à adopter une approche prudente, qui consiste à comparer les résultats de simulations de plusieurs modèles afin de vérifier la validité des prévisions. Ce travail a permis d'identifier des forces et des faiblesses pour chacun des modèles, ce qui permettra dorienter le développement de ces outils dans le futur.

\section{Remerciements}

Ces travaux ont été réalisés dans le cadre du projet interne $\mathrm{n}^{\mathrm{o}} 142332123$ de la Direction de la recherche forestière (DRF). Les auteurs remercient Madame Denise Tousignant de la DRF pour la révision linguistique du manuscrit, Messieurs François Guillemette et Filip Havreljuk de la DRF pour leurs commentaires sur les versions précédentes du manuscrit et Monsieur Jean Noël de la DRF pour la Fig. 1. Les auteurs tiennent également à remercier les deux réviseurs anonymes qui ont révisé le présent travail.

\section{Références}

Auger, I. 2017a. Comparaison des estimations des modèles de croissance forestière Natura-2009 et Natura-2014 sur une période de 30 ans. Gouvernement du Québec, ministère des Forêts, de la Faune et des Parcs, Direction de la recherche forestière. Note de recherche forestière $\mathrm{n}^{0} 147,31$ p. Disponible à http://www.mffp.gouv.qc.ca/ publications/forets/connaissances/recherche/Auger-Isabelle/ Note147.pdf [consulté le 29 novembre 2017].

Auger, I. 2017b. Guide d'utilisation du simulateur de croissance forestière Natura-2014 sur Capsis (version 1.0). Guide du gouvernement du Québec, ministère des forêts de la faune et des parcs, Direction de la recherche forestière. Disponible à https://www.mffp. gouv.qc.ca/publications/forets/connaissances/recherche/AugerIsabelle/GuideUtilisationNatura-2014.pdf [consulté le 14 septembre 2017].

Burkhart, H. E. 2003. Suggestions for choosing an appropriate level of modelling for forest stands. Dans Modelling forest systems. pp. 3-10 CAB International publishing, Wallingford, U.K.

Boucher, D., L. De Grandpré et S. Gauthier. 2003. Développement d'un outil de classification de la structure des peuplements et comparaison de deux territoires de la pessière à mousse du Québec. Forest. Chron. 79 : 318-328. Doi: 10.5558/tfc79318-2.

Dufour-Kowalski, S., B. Courbaud, P. Dreyfus, C. Meredieu et F. de Coligny. 2012. Capsis: an open software framework and community for forest growth modelling. Annals of Forest Science 69: 221-233.

Fortin, M. et L. Langevin. 2010. Artémis-2009 : un modèle de croissance basé sur une approche par tiges individuelles pour les forêts du Québec. Gouvernement du Québec, ministère des Ressources naturelles et de la Faune, Direction de la recherche forestière. Mémoire de recherche forestière $\mathrm{n}^{0} 156$. Disponible à https://www.mffp. gouv.qc.ca/publications/forêts/connaissances/recherche/FortinMathieu/Mémoire 156.pdf [consulté le 14 septembre 2017].

Garet, J. 2008. Influence des caractéristiques de la sénescence sur la possibilité forestière. Thèse $\mathrm{M}$. Sc., Département des sciences du bois et de la forêt, Université Laval, Québec, Qc. Disponible à http:// theses.ulaval.ca/archimede/fichiers/25643/25643.html [consulté le 28 novembre 2017].

Gray, D.R., J. Régnière et B. Boulet. 2000. Analysis and use of historical patterns of spruce budworm defoliation to forecast outbreak patterns in Quebec. For. Ecol. Manage. 127:217-231. doi: 10.1016/ S0378-1127(99)00134-6.

Groot, A., S. Gauthier et Y. Bergeron. 2004. Stand dynamics modelling approaches for multicohort management of eastern Canadian boreal forests. Silva Fennica. 38:437-448. doi: 10.14214/sf.411.

Härkonen, S., A. Mäkinen, T. Tukola, J. Rasinmäki et J. Kalliovirta. 2010. Evaluation of forest growth simulators with NFI permanent sample plots from Finland. For. Ecol. Manag. 259:573-582. doi: 10.1016/j.foreco.2009.11.015.

Landsberg, J. J. 2003. Modelling forest ecosystems: State of the art, challenges and futur directions. Can. J. For. Res. 33:385-397. doi: 10.1139/x02-129.

Larocque, G. R., D. Mailly et M. Gaudreault. 2009. Forest modelling in Quebec: Context, challenges and perspectives. Forest. Chron. 85:702-705. doi: 10.5558/tfc85702-5.

MacLean, D. A. et A. R. Andersen. 2008. Impact of a spruce budworm outbreak in balsam fir and subsequent stand development over a 40-year period. Forest. Chron. 84:60-69. doi: 10.5558/tfc84060-1. Mäkinen, A., A. Kangas, J. Kalliovirta, J. Rasinmäki et E. Välinmäki. 2008. Comparison of treewise and standwise forest simulators by mean of quantile regression. For. Ecol. Manag. 255:2709-2717. doi: 10.1016/j.foreco.2008.01.048.

McCullagh, A., K. Black et M. Nieuwenhuis. 2017. Evaluation of tree and stand-level growth models using national forest inventory data. Eur. J. For. Res. Doi: 0.1007/s10342-017-1025-8.

MFFP. 2014. [Ministère des Forêts, de la Faune et des Parcs]. Réseaux des placettes-échantillons permanentes du Québec méri- 
dional. Gouvernement du Québec, ministère des Forêts, de la Faune et des Parcs, Direction des inventaires forestiers. 10 p. Disponible à : https://mffp.gouv.qc.ca/publications/forets/connaissances/ReseauxPEP.pdf [consulté le 29 novembre 2017].

MRN. 2013. [Ministère des Ressources naturelles]. Le guide sylvicole du Québec, Tome 1, Les fondements biologiques de la sylviculture. Ouvrage collectif sous la supervision de B. Boulet et M. Huot, Les Publications du Québec, Québec, Qc. 1044 p.

Porté, A. et H. H. Bartelink. 2002. Modelling mixed forest growth : A review of models for forest management. Ecol. Model. 150 141-188. doi : 10.1016/S0304-3800 (01) 00476-8.

Pothier, D. et D. Mailly. 2006. Stand-level prediction of balsam fir mortality in relation to spruce budworm defoliation. Can. J. For. Res. 36: 1631-1640. doi:10.1139/x06-062.

Pothier, D. et I. Auger. 2011. Natura-2009 : un modèle de prévision de la croissance à léchelle du peuplement pour les forêts du Québec. Gouvernement du Québec, ministère des Ressources naturelles et de la Faune, Direction de la recherche forestière. Mémoire de recherche forestière $\mathrm{n}^{0} 163.56$ p. Disponible à : www.mffp.gouv.qc.ca/publications/forêts/connaissances/recherche/Auger-Isabelle/Memoire163 .pdf [consulté le 17 septembre 2017].

Poulin, J. 2013a. Cartographie. Fascicule 2.1. Dans Bureau du forestier en chef. Manuel de détermination des possibilités forestières 2013-2018. Gouvernement du Québec, Roberval, Qc., p.43-45. Disponible à : forestierenchef.gouv.qc.ca/wp-content/uploads/2013/01/ MDPF_VF.pdf [consulté le 14 septembre 2017].

Poulin, J. 2013b. Strates d’aménagement. Fascicule 2.2. Dans Bureau du forestier en chef. Manuel de détermination des possibilités forestières 2013-2018. Gouvernement du Québec, Roberval, Qc., p.47-50. Disponible à : forestierenchef.gouv.qc.ca/wp-content/ uploads/2013/01/MDPF_VF.pdf [consulté le 14 septembre 2017].
Power, H. 2016. Comparaison des biais et précision des estimations de surface terrière totale, avec et sans coupe partielle, des modèles Artémis-2009 et Artémis 2014 sur une période de 40 ans. Gouvernement du Québec, ministère des Forêts, de la Faune et des Parcs, Direction de la recherche forestière. Note de recherche forestière $n^{0} 143,21$ p. Disponible à : http://www.mffp.gouv.qc.ca/publications/ forets/connaissances/recherche/Power-Hugues/Note143.pdf [consulté le 14 septembre 2017].

Pretzsch, H., R. Grote, B. Reineking, T.H. Rötzer et S.T. Siefert. 2008. Models for forest ecosystem management: An European perspective. Ann. Bot. 101: 1065-1087. doi:10.1093/aob/mcm246.

Stage, A.R. 2003. How forest models are connected to reality: Evaluation criteria for their use in decision support. Can. J. For. Res. 33: 410-421. doi: https://doi.org/10.1139/x02-203.

Vanclay, J.K. et J.P. Skovsgaard. 1997. Evaluating forest growth models. Ecol. Model. 98:1-12. doi: 10.1016/S0304-3800(96)01932-1. van Oijen, M. et al. 2013. Bayesian calibration, comparison and averaging of six forest models, using data from Scots pine stands across Europe. For. Ecol. Manage. 289: 255-268. doi:10.1016/ j.foreco.2012.09.043.

von Gadow, K. et G. Hui. 1999. Modelling forest development (Vol. 57). Springer Science \& Business Media. Dordrecht, Nederland. 213 p. doi: 10.1007/978-94-011-4816-0.

Vospernick, S., R. A. Monserud et H. Sterba. 2015. Comparing individual tree growth models using principle of stand growth for Norway spruce, Scots pine and European beech. Can. J. For. 45:1006-1018.doi: 10.1139/cjfr-2014-0394

Yue, C., U. Kohlne et S. Hein. 2008. Combining tree and stand level models: A new approach to growth prediction. For. Sci. 54:553-566. 